\title{
La huella de la crisis y la estrategia de supervivencia en la industria hotelera Española: Una aproximación a través de los estados financieros
}

\author{
Crisis and survival strategy in the hospitality industry: An approach through financial statements
}

\author{
Remedios Ramón-Dangla
}

Universidad de Alicante, Facultad de CC. Económicas y Empresariales, Departamento de Economía Financiera y Contabilidad. Carretera de San Vicente del Raspeig s/n. 03690, Alicante, España, remedios.ramon@ua.es

\begin{abstract}
Resumen
El objetivo del trabajo es ver si la estrategia de supervivencia de los hoteles españoles a la actual crisis ha dejado huella en sus Estados Financieros y si dichos cambios se han producido al mismo ritmo que evolucionaba la economía nacional. Para ello, se ha obtenido una muestra de 975 empresas de la base $\mathrm{SABI}$, se han elaborado algunos ratios financieros y se les han aplicado una prueba t de mediciones apareadas. Se ha rechazado la hipótesis nula de igualdad de medias con una confianza del $95 \%$ para los ratios de rentabilidad, solvencia y endeudamiento: La crisis puede estar en la base de unos valores peores de rentabilidad pero mejores de solvencia, endeudamiento, liquidez y eficiencia. Por otra parte, mediante regresión estadística se han buscado las variables económicas que mejor explican la evolución de los ingresos netos en el conjunto de hoteles: la llegada de turistas extranjeros es la base para el comportamiento anti-cíclico del sector.
\end{abstract}

Palabras Clave: Industria hotelera, estados financieros, solvencia, endeudamiento, rentabilidad, crisis económica.

\section{Abstract}

The aim of this study is to see if the survival strategy of Spanish Hotels on the current crisis has left a mark in their Financial Statements and if the changes have taken place at the same time as the national economy was evolving. To do this, we have obtained a sample of 975 companies from SABI. We have made the financial ratios and applied them a paired t-test measurements. It has rejected the null hypothesis of equal means with a 95\% confidence for profitability, solvency and indebtedness ratios: the crisis could have been the reason of lower profitability and debt and higher levels of liquidity, solvency and efficiency. On the other hand, with a regression we have researched the economic variables which can explain better the hotels income evolution: the foreign tourist arrivals are the key for the anti-cyclical behavior of the sector.

Keywords: Hospitality industry, financial statements, solvency, indebtedness, profitability, economic crisis.

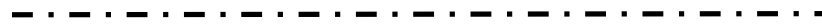

\section{Introducción}

El sector turístico es uno de los más importantes en cualquier economía tanto por su capacidad de generar empleo y riqueza como por sus efectos sobre la balanza de pagos. Según el World Travel and Tourism Council (WTTC 2014) este sector creó en 2014 más de 101 millones de empleos y generó el 9,5\% del PIB mundial. Además, la (OMT, 2004a y 2010) confirman que, desde 1994, el turismo ha sido la principal fuente de entrada de divisas en los países más atrasados (excluida la industria petrolera) y un pilar clave para la economía de la Unión Europea, donde el sector representa más de un $10 \%$ del PIB y de un $12 \%$ del empleo.

En España, el turismo ha demostrado ser un sector estable y resistente a las crisis y así lo recogen entre muchos Torres (1994), Massieu (2004), Torres, Ramírez y Rodríguez (2014), Aurioles (2011) o Ramón (2002) ó el Observatorio de la Industria Hotelera Española, por lo que su tratamiento se convierte en un elemento clave para la economía nacional. La tradicional importancia del turismo en España ha favorecido que nuestras empresas estén altamente especializadas y sean muy competitivas internacionalmente, fundamentalmente, las hoteleras. Así, en 2013, de entre las 40 cadenas hoteleras más grandes del mundo, 5 fueran españolas (hotelsmag, 325, 2013).

La importancia tanto por número como por volumen y tamaño de las empresas hoteleras españolas ha propiciado su estudio desde distintas vertientes económicas. Desde el lado de la sostenibilidad Pérez y Rodríguez (2015) hacen hincapié en la necesidad de mejorar la comunicación de la Responsabilidad Social Corporativa (RSC) de los hoteles en aras de mantener la competitividad internacional. Más concretos son Fernández y Cuadrado (2011) al proponer un estudio en materia de RSC centrado en una política integral del sector y no solo en algunas medidas. Por otra parte, Souto (2015) abunda en la importancia de la utilización de las nuevas tecnologías y las redes sociales para no ceder clientes y mantener el rendimiento económico en un sector donde cada vez hay más competidores internacionales o Murphy, Chen y Cossutta (2015) hacen hincapié en la necesidad de innovar en las distintos formas de reserva para conseguir distintos tipos de clientes y Fernández y Becerra (2013) determinan la fuerte relación entre la calidad, medida mediante la inversión en intangibles, y la eficiencia del hotel, sobre todo, para grandes cadenas y hoteles resort. En este sentido y atendiendo a la búsqueda del retorno económico García y Armas (2007) concluyen que la fidelización de clientes pero sobre todo, el desarrollo de unos hábitos sostenibles en materia medioambiental son fundamentales para mantener una oferta diferenciada que optimice la rentabilidad de las empresas hoteleras.

Pero a pesar de la abundante literatura económica sobre el sector, apenas existen estudios sobre la marcha de los estados financieros y de cómo las empresas están afrontando la crisis. La evolución de ratios de rentabilidad económica, de solvencia 0 de endeudamiento nos pueden estar diciendo de qué manera las empresas están sobreviviendo, lo que complementaría la visión que ya se está haciendo del sector por otras ramas de la economía. El objetivo de este trabajo es por tanto, analizar el impacto de la crisis sobre la industria hotelera española medido a través de los estados contables-financieros de las empresas y ver si el sector evoluciona en sintonía con las variables macro-económicas.

La innovación de los procesos productivos de los hoteles es uno de los factores principales de supervivencia empresarial, tal y como indican autores como Martínez (2009) o Vila, Enz y Costa (2012). La modernización y el desarrollo de nuevos modelos de gestión 
permiten mejorar la rentabilidad empresarial según Pertusa, Tari, Pereira, Molina y López (2103), quienes consiguen establecer una relación positiva entre las certificaciones de calidad y el desarrollo de gestiones sostenibles llevada a cabo por empresas hoteleras y su rentabilidad medida a través del RevPAR (Revenue per Available Room o ingresos por habitación disponible) o del ADR (Average Daily Rate. Tarifa media diaria o facturación media por habitación ocupada), lo que coincide con otros estudios previos tanto en el ámbito de las empresas hoteleras Rubio, Alonso y Rodriguez (2011) o el de Álvarez, Faiz y Del Río Rama (2012) como en otros ámbitos o sectores económicos estudiados por otros autores como Chow-Chua, Goh y Wan (2003) o por Mak (2011).

Sin embargo, en menos ocasiones se ha llevado a cabo un análisis económico-financiero en el conjunto de hoteles españoles para ver el impacto de la crisis. Trabajos como el de González y Jareño (2014) analizan los cambios entre fondos propios y financiación ajena en las grandes cadenas hoteleras durante la crisis y concluyen en que ha habido un importante intercambio de fuente de financiación ajena por propia en la mayor parte de empresas. Por otro lado, Lado-Sestayo, Otero-González, Vivel-Bua y Martorell-Cunill (2016) analizan los motivos de las quiebras en hoteles españoles desde una perspectiva financiera. Estos autores determinan que las variables financieras vinculadas a la rentabilidad, endeudamiento, estructura económica y actividad junto con la antigüedad y tamaño del hotel tienen incidencia sobre la probabilidad de quiebra en un periodo de crecimiento económico. Por su lado, Gémar, Moniche y Morales (2016) determinan que, en un contexto de crecimiento económico, las variables no financieras como el tamaño, la localización o la administración influyen más sobre la ratio de supervivencia, lo que va en sintonía con lo analizado por Tsai, Song y Wong (2009). Sin embargo, en ninguno de los casos, se analiza el efecto de la actual crisis en el conjunto del sector. De ahí, nuestro interés en analizar los estados financieros de las empresas hoteleras en un periodo de crisis y de ver qué variables macro-económicas explicarían mejor su evolución.

El análisis de la información financiera de las empresas obedece a diversas finalidades económico-contables y por tanto, se centra en distintos aspectos de la información financiera y de su calidad, tal y como explican Sánchez (2002), Godoy (2004), Gonzalo, Pérez y Serrano (2000). Para el trabajo que nos ocupa nos centraremos en dos aspectos. Por una parte, en un análisis económico, entendiendo como tal, el análisis de la eficacia, de la eficiencia y de la productividad empresarial estudiado a través de la actividad comercial y de la rentabilidad de los capitales invertidos. Y por otra parte, en un análisis financiero, para ver el grado de liquidez que presentan los elementos activos y la mayor o menor reembolsabilidad de las masas de pasivo: la autonomía financiera y la solvencia.

Siguiendo a Archel, Lizárraga y Sanchez (2008), toda asignación de recursos conlleva un riesgo, pero también determina cierta rentabilidad. Así, el objetivo del análisis de los estados contables consiste en someter la información a un estudio que ayude a los usuarios a tomar las decisiones financieras racionalmente como explican Amat (2000), Esteo (1998) o Gonzalo et al. (2000). Continuando con el mismo autor, la comparación inter-empresas se puede efectuar tomando como referente una muestra representativa de empresas del sector o cualquier conjunto de empresas a las que se pretenda comparar. Para este acometido resultarán de gran utilidad los ratios elaborados mediante cocientes de dos magnitudes entre las que exista una relación económico-financiera. De este modo, tal y como indican Garrido e Íñiguez (2012), se medirán algunos aspectos de las empresas y se podrán hacer comparaciones entre distintos años y empresas. No obstante, como explican Archel et al. (2008), el estudio de los ratios no facilita datos sobre las causas de los cambios operados en las empresas. Por esta razón, es necesario estudiar las empresas dentro de la coyuntura económica y ver aquellas variables económicas que mejor explican su evolución contable. De ahí que dividamos nuestro trabajo en este primer punto de introducción. Un segundo punto de contextualización económico y descripción de nuestras hipótesis, el tercero explicaría la metodológica, en un cuarto punto, analizaríamos los resultados y acabaríamos con unas conclusiones.

\section{Crisis económica y sector hotelero. Formulación de hipótesis}

No cabe duda de que cualquier crisis económica afecta a la rentabilidad, eficacia, solvencia y eficiencia empresarial pero además, en el caso de empresas vinculadas al sector turístico, su situación podría ser más vulnerable ya que a las crisis de origen económico, habría que añadir las crisis de seguridad, tal y como subrayan Aurioles (2011) o Torres et al. (2014). Para estos autores los conflictos bélicos, los atentados o las alarmas sanitarias pueden reducir los deseos de viaje, y por tanto, generar un colapso turístico, como puso de manifiesto, por ejemplo Saharpley (2008) al analizar el efecto del Tsunami sobre el sector turístico en el área Asiática, o como describieron Lo, Cheung y Law (2007) respecto a los efectos que sobre la industria hotelera de Hong Kong trajo la aparición de varios casos de SARS (Síndrome Respiratorio Agudo Grave) en China, aunque también siguen existiendo un sector de consumidores menos afectados por estas crisis y que siguen demando destinos afectos por riesgo como indican Hajibaba, Gretzel, Leisch, Dolnicar (2015).

En este sentido, autores como Aurioles (2011), Torres et al. (2014) o Ruíz y Casado (2011) coinciden en que el 2009 fue uno de los peores años del turismo internacional por causas económicas, sobre todo, para algunas actividades dentro del sector como pueden ser los viajes de negocios, el transporte aéreo o los hoteles.

La crisis económica iniciada en nuestro país durante el segundo trimestre de 2008 y que afectó de manera negativa a indicadores económicos como el PIB, el consumo interno o el desempleo Ortega y Peñasola (2012), trajo un descenso del turismo nacional e internacional. No obstante, la evolución de la demanda hotelera o del número de pernoctaciones estudiado por autores como Torres et al. (2014), Ruíz y Casado (2011) ó Aurioles (2011), ponen de manifiesto que, para la industria hotelera, la crisis fue intensa pero corta, ya que 2010 fue el año de la recuperación.

Si bien, son abundantes los estudios que relacionan las estrategias y capacidad de la industria hotelera para adaptarse a los distintas situaciones económicas y de crisis, entre muchos, Jiung-Bin, MuChen y Ling-Feng (2013) Ramón (2002), Aurioles (2011), Torres (1994), Dahles, y Susilowati (2015) y Gónzalez y Jareño (2014), Cohen y Neal (2010), Ruíz y Casado (2011), Massieu (2004) Lado- 
Sestayo et al. (2016), no lo son tanto aquellos que focalizan el estudio en el análisis de los estados contables de las empresas hoteleras $y$, aunque el crecimiento del número de turistas y pernoctaciones nos pone sobre la pista de la recuperación del sector, esta variable por sí sola no es una garantía de mejora de la rentabilidad empresarial y por tanto, de supervivencia a largo plazo. En efecto, las empresas para ser viables a futuro no solo han de tener un gran volumen de negocio sino que éste debe ser rentable, lo que nos lleva a plantearnos nuestra primera hipótesis.

H1: A pesar de que las pernoctaciones empiezan a recuperarse a partir de 2010, los efectos de la crisis han sido tan intensos y la industria hotelera ha necesitado contener tanto los precios que, en 2013, el número de pernoctaciones alcanzó el de pre-crisis, pero las rentabilidades económicas no.

Por otra parte, sabemos que la demanda hotelera depende de múltiples factores externos o autónomos como los hábitos de consumo, las modas, la seguridad, los turoperadores, los precios y también, del nivel de renta. Lo que se podría representar por la ecuación (1)

\section{$D=D_{0}+D_{1} Y_{d}$}

$D_{0}$ sería la demanda autónoma o externa, la que no depende de la renta, $\left(D_{0}>0\right)$

$D_{1}$ Sería la propensión marginal a consumir. Mide el efecto de una unidad monetaria adicional de renta disponible sobre el consumo $\left(0<D_{1}<1\right)$

$Y_{d}$ es la renta disponible una vez pagados los impuestos: Renta Neta Disponible. Variaciones en el nivel de renta disponible, afectan en el mismo sentido a la demanda, lo que se conoce como elasticidad demanda renta positiva.

$$
\varepsilon \mathrm{y}_{d}=\frac{\text { variación porcentual cantidad demandada }}{\text { variación porcentual renta }}>0
$$

En este sentido Iranzo (2003) determinan que la demanda turística es elástica respecto a la renta: conforme aumenta la renta disponible, aumenta la demanda. De ahí que los servicios turísticos, en general y los hoteleros en particular, sean servicios normales y por tanto, tengan una elasticidad demanda-renta positiva. Sin embargo, en el caso de España, a pesar de la fuerte contracción en la renta neta disponible o del PIB, la demanda de alojamiento hotelero no se vio tan mermada. Además, a partir de 2010, cuando se agudiza la crisis en España, los ingresos netos de los hoteles empezaron a recuperarse. La llegada de turistas internacionales procedentes fundamentalmente de Inglaterra y Alemania, donde la crisis era menos intensa, podría estar en la base de este comportamiento. De ahí que formulemos nuestra segunda hipótesis

H2: Aunque la demanda hotelera mantiene una elasticidad demanda-renta positiva, durante este periodo de crisis, ha seguido una evolución dispar a la de la renta neta disponible o el PIB contrayéndose en menor medida. El sector hotelero ha actuado de manera anti-cíclica para la economía nacional

La importancia social y económica de la actividad turística en España pone al sector hotelero en el centro de los análisis. Según el Instituto Nacional de Estadística (INE), el turismo aporta al PIB nacional más de un $10 \%$ y genera más de 5 millones de empleos.
El número de turistas internacionales que llegaron a España en 2013 fue de más de 41 millones, un 4,8\% por encima de la cifra alcanzada en el ejercicio anterior y con un crecimiento continuado desde 2010. Sin embargo, la rápida recuperación de la demanda del sector y su relevancia no fue eximente para que las empresas hoteleras españolas se vieran afectadas por la restricción del crédito que afectaba al mundo.

Existen innumerables estudios que demuestran que el endeudamiento empresarial está estrechamente relacionado con la evolución de variables macroeconómicas. En este sentido, destacamos a Jordà, Schularik y Taylor (2011) quienes, después de analizar las recesiones económicas entre 1870 y 2008 en catorce países desarrollados, relacionan el endeudamiento de las empresas y la inestabilidad de la economía, concluyendo que existe una clara relación entre el apalancamiento financiero y la dureza que presentan las etapas de recesión posteriores.

En el caso español, es necesario considerar las consecuencias que se derivaron del ingreso en el euro y que coincide con el patrón de comportamiento descrito por Fernández (2010) cuando indica que la política de bajos tipos de interés inundó el mercado con un exceso de liquidez que estimuló a las familias, empresas y bancos a endeudarse. La diferencia en las preferencias por el endeudamiento de las empresas españolas respecto de sus vecinos viene avalada por la tesis de Rajan y Zingales (1995); Demirgüç-Kunt y Maksimovic (1999); Booth, Aivazian, DermirgucKunt y Maksimovic (2001); Hanousek y Shamshur (2011) quienes defienden el argumento de que los costes y los beneficios que las empresas tienen en cuenta en sus decisiones de estructura de capital no solo vienen determinados por sus características específicas sino también por las peculiaridades del país o, como indican Gill de Albornoz y Giner (2013), por el tipo de sector en el que desarrollen su actividad.

Rubio y Sogorb (2011) concluyen que las empresas españolas, en contradicción con la evidencia empírica internacional y con los estudios precedentes de Miguel y Pindado (2001) y González y González (2008), se mueven con mayor rapidez hacia ratios objetivos de endeudamiento en las fases contractivas del ciclo económico que en las expansivas, por tanto, la caída del PIB y la fuerte reducción de la demanda interna han tenido que provocar cambios en las cuentas empresariales de nuestras empresas objeto de estudio que las habrá llevado a reducir sus pasivos.

La contracción del crédito y su encarecimiento real provocó que las empresas que antes habían tenido un fuerte nivel de endeudamiento intentaran desapalancarse. Las bajas tasas de beneficio empresarial dificultaban la devolución de los créditos y evidenciaban el sobredimensionamiento del endeudamiento, lo que iba a llevar ineludiblemente, a una restructuración de los pasivos contables en las empresas. La estructura financiera empresarial debía cambiar, incluso en un sector, que parecía haber sufrido la crisis durante solo un par de años, lo que nos lleva a formular nuestra tercera hipótesis.

H3: A pesar de que el volumen de negocio de las empresas hoteleras parece que se haya contraído menos que la economía española durante la crisis, la dificultad para acceder al crédito y su encarecimiento ha originado un efecto de desapalancamiento y de recapitalización de los fondos propios. 


\section{Descripción de variables, muestra y metodología}

El objetivo de este trabajo es ver, a través de los Estados Financieros de los hoteles españoles, si la estrategia de supervivencia sectorial a la actual crisis ha dejado huella y si los cambios se han producido al mismo ritmo que evolucionaba la economía nacional. Para ello, sobre la población total de 2.773 hoteles que nos da la base de datos SABI (Sistema de Análisis de Balances Ibéricos) hemos aplicado 3 filtros en cascada. Primero eliminamos las sociedades que no habían estado activas durante todo el periodo estudiado (2007-2013), ya que nuestro objetivo era precisamente, analizar el antes y el después de la crisis en los resultados y la estructura económica-financiera de los hoteles. Después, aplicamos la Directiva Europea 2013/34/UE del Parlamento Europeo y del Consejo de 26 de junio de 2013, sobre los estados financieros anuales y consolidados de las empresas para desechar las micro-pyme: Total Activo (TA) inferior a $350.000 €$ y una Cifra Neta de Negocio (CNN) inferior a 700.000€. Por último, excluimos las empresas dependientes de su matriz y que consolidan con ésta. Así, nuestra muestra depurada quedó en 975 empresas y sobre ella, comprobamos que su tamaño fuese representativo de la población original con una seguridad del $99 \%$, con un margen de error del $3 \%$ y una proporción esperada del $50 \%$.

Posteriormente y para el análisis, definimos el conjunto de ratios financieros para medir las dimensiones económicas clave de la actividad de una empresa siguiendo a Foster, (1986); Rivero (1996), Gonzalo et al. (2000), Garrido e Î́niguez (2008), Archel et al. (2008) Amat (1994), Urías (1993), Esteo (1998) y que son, además de su estructura económica, su rentabilidad, el apalancamiento financiero, la solvencia, la liquidez y la operatividad o eficiencia (Tabla1).

Tabla 1 - Definición de variables

\begin{tabular}{|c|c|c|}
\hline \multirow{4}{*}{ Rentabilidad } & RE1 & Resultado Explotación/Activo Total \\
\hline & RE2 & Resultado Explotación/Cifra Neta Negocio \\
\hline & RE3 & Resultado Ejercicio/Patrimonio Neto \\
\hline & RE4 & Resultado Antes de Impuestos e Intereses/Activo Total \\
\hline \multirow{6}{*}{$\begin{array}{l}\text { Endeudamiento } \\
\text { o Solvencia }\end{array}$} & Rend1 & Activo Total/Pasivo Total \\
\hline & Rend3 & Pasivo no Corriente/Activo Total \\
\hline & Rend4 & Resultado de Explotación/Gastos Financieros \\
\hline & Rend5 & Gastos Financieros/Total Pasivo \\
\hline & Rend6 & Gastos Financiero/Resultado Antes de Impuestos e Intereses \\
\hline & Rend7 & Gastos Financieros/Cifra Neta de Negocio \\
\hline $\begin{array}{l}\text { Equilibrio económico- } \\
\text { financiero }\end{array}$ & EQ4 & (Pasivo no Corriente + Patrimonio Neto)/Activo no Corriente \\
\hline \multirow{3}{*}{ Estructura Económica } & EE1 & Activo Corriente/Activo Total \\
\hline & EE2 & Activo no Corriente/Activo Total \\
\hline & EE3 & Tesorería/Activo Total \\
\hline \multirow{3}{*}{ Liquidez } & L1 & Activo Corriente/Pasivo Corriente \\
\hline & L2 & (Activo Corriente-Pasivo Corriente)/Activo Total \\
\hline & L3 & (Activo Corriente-Existencias)/Pasivo Corriente \\
\hline \multirow{2}{*}{$\begin{array}{l}\text { Operatividad o } \\
\text { Eficiencia }\end{array}$} & 01 & (Activo Corriente-Pasivo Corriente)/Cifra Neta de Negocios \\
\hline & $\mathrm{O} 2$ & Cifra Neta de Negocios/Activo Total \\
\hline
\end{tabular}

Fuente: Elaboración propia.

Tal y como ya dijo Lev (1969), el análisis empírico no debe incorporar simultáneamente la totalidad de ratios financieros debido a los problemas de especificación, multicolinealidad y redundancia informativa, por tanto, siguiendo a multitud de autores posteriores a Lev (1969), entre los que citamos a Lee y Wu (1988), Peles y Schneller (1989), Wu y Ho (1997), Gallizo y Salvador (1997), rechazamos aquellos ratios que mostraban una alta correlación entre sí: coeficiente de correlación de Pearson mayor al $40 \%$ y que no cumplían con la normalidad y la homogeneidad de las varianzas como lo han hecho antes Altmant (1968), Altmant y Sabato (2007) o Gill de Albornoz y Giner (2013).

De este modo, los ratios seleccionados y que serán utilizados en el análisis empírico posterior son: RE2, RE4, Rend1, EQ3, EE1, L1, O1y O3.

Finalmente, se comprobó si habían existido cambios en las medias muestrales de cada uno de los ratios seleccionados antes y después de la crisis mediante una prueba T-Student para muestras apareadas, con un intervalo de confianza del 95\% y asumiendo igualdad de varianzas siguiendo a Sawilowsky (2005). A través del estadístico t y del p-valor en la prueba T-Student, se contrastó la hipótesis nula de igualdad de medias para cada grupo de ratios en el periodo objeto de estudio y se vio que las empresas hoteleras que habían sobrevivido al "shock económico", en general, presentaban valores más bajos de rentabilidad y endeudamiento y mejores niveles de liquidez, solvencia y eficiencia que antes de la crisis.

Por otra parte y para comprobar la relación y dependencia de la industria hotelera con otras variables macroeconómicas, construimos un modelo de dependencia basado en Regresiones Lineales Múltiple (RLM) (3). La elección del modelo RLM no solo se debía a su amplia aplicación para describir y predecir el comportamiento de una variable cuantitativa a partir de los 
valores de otras variables explicativas sino que también estaba condicionado por la disponibilidad de datos.

$$
C N N_{i}=\alpha+\beta_{1} P I B_{p m i}+\beta_{2} Y_{d i}+\beta_{3} V_{e x i}+\beta_{4} V_{e i}+\varepsilon
$$

Donde la variable dependiente: $\mathrm{CNN}_{i}$ es la Media de la Cifra Neta de Negocio de nuestra muestra de hoteles para cada año i.

Consideramos $\mathrm{CNN}_{\mathrm{i}}$ como dependiente por ser una variable clave de la cuenta de pérdidas y ganancias y que indica el volumen de negocio de la empresa, ya que se compone de los importes de la venta de los productos o de la prestación de servicios correspondientes a las actividades ordinarias, deducidas las bonificaciones y demás reducciones sobre las ventas, así como el impuesto sobre el valor añadido y otros impuestos directamente relacionados con la mencionada cifra de negocios.

$\mathrm{PIB}_{\text {pmi }}$ Es el Producto Interior Bruto a precios de mercado para el año i $\mathrm{Y}_{\mathrm{di}}$ Es la Renta Disponible Neta por Habitante para cada año i (Renta per Cápita)

$V_{\text {exi }}$ Es el número de Viajeros Extranjeros en cada año $i$

$V_{\text {ei }}$ Es el número de Viajeros Españoles en cada año $\mathrm{i}$

Cada $\beta$ representa la importancia relativa que tiene cada una de las variables en la explicación de la Cifra Neta de Negocio con un intervalo de confianza del $95 \%$

$\varepsilon$ Es la perturbación aleatoria
El método utilizado para aproximar el modelo (RLM) fue el método "hacia atrás". En él se introducen todas las variables en la ecuación y después se van excluyendo una tras otra mientras satisfagan el criterio de eliminación basado en la baja correlación parcial con la variable dependiente. Previamente, comprobamos la existencia de un alto nivel de correlación parcial entre el $\mathrm{PIB}_{\mathrm{pmi}}$ y la $\mathrm{Y}_{\mathrm{d}}$ representado por un coeficiente de correlación de Pearson de 0,875, lo que nos arrastraba a eliminar, de entre las dos variables, aquella que explicara peor nuestra variable dependiente, esto es, el PIB pmi.. $_{\text {. }}$

EI modelo fue desarrollado mediante SPSS21, después de comprobar que, por un lado, se cumplían las hipótesis de normalidad del término de error y de linealidad de la variable respuesta frente a las predictivas y que, por otro lado, el modelo ofrecía un alto poder explicativo representado por un "Rcuadrado" y "R-cuadro corregida" próximo a 1. Del ajuste de la regresión lineal hemos podido aproximar cuáles son las macromagnitudes que mayor incidencia tienen en la evolución de la Cifra Neta de Negocio de la industria hotelera.

\section{Resultados y análisis}

La Tabla 2 presenta los estadísticos descriptivos de las ratios económico-contable de las empresas hoteleras. A priori, se puede intuir que sí que se han producido cambios significativos en ellos como consecuencia de la crisis.

Tabla 2 - Estadísticos descriptivos

\begin{tabular}{|l|c|c|c|c|c|}
\hline & N & Mínimo & Máximo & Media & Desv. típ. \\
\hline RE2_13 & 975 & $-5,269$ & 2,629 & 0,015 & 0,360 \\
\hline RE2_07 & 975 & $-3,999$ & 2,576 & 0,086 & 0,257 \\
\hline RE4_13 & 975 & $-3,229$ & 0,684 & 0,016 & 0,163 \\
\hline RE4_07 & 975 & $-2,408$ & 0,682 & 0,056 & 0,112 \\
\hline Rend1_13 & 975 & 0,096 & 35,322 & 2,990 & 3,465 \\
\hline Rend1_07 & 975 & 0,201 & 30,662 & 2,716 & 2,937 \\
\hline EQ3_13 & 975 & $-89,367$ & 252,426 & 2,218 & 13,860 \\
\hline EQ3_07 & 975 & $-287,337$ & 287,856 & 3,020 & 21,430 \\
\hline EE1_13 & 975 & 0,003 & 1,009 & 0,208 & 0,206 \\
\hline EE1_07 & 975 & 0,006 & 0,980 & 0,230 & 0,203 \\
\hline L1_13 & 975 & 0,015 & 29,115 & 1,708 & 2,572 \\
\hline L1_07 & 975 & 0,016 & 22,070 & 1,618 & 2,124 \\
\hline O1_13 & 975 & $-15,979$ & 8,584 & $-0,051$ & 1,048 \\
\hline 01_07 & 975 & $-5,300$ & 4,343 & $-0,042$ & 0,805 \\
\hline O3_13 & 975 & 0,001 & 1,227 & 0,379 & 0,118 \\
\hline O3_07 & 975 & 0,005 & 1,463 & 0,353 & 0,117 \\
\hline
\end{tabular}

Fuente: Elaboración propia.

El análisis de diferencia de medias recogido en la tabla 3 muestra unos resultados en línea con el estudio de descriptivos previos. En general, todos los resultados han mostrado diferencias significativas entre el antes y el después de la crisis, lo que nos lleva a rechazar la hipótesis nula de igualdad de medias para todos los ratios estudiados excepto para la liquidez (L1) y el capital corriente o fondo de maniobra (01).

Tabla 3 - Prueba t-student de muestras relacionadas

\begin{tabular}{|l|c|c|c|c|}
\hline & \multicolumn{3}{|c|}{ 95\% Intervalo confianza para diferencia } \\
\hline \multicolumn{1}{|c|}{ Par } & Media & Inferior & Superior & p-valor \\
\hline RE2_13-RE2_07 & $-0,071$ & $-0,096$ & $-0,045$ & 0,000 \\
\hline RE4_13-RE4_07 & $-0,039$ & $-0,051$ & $-0,028$ & 0,000 \\
\hline Rend1_13-Rend1_07 & 0,274 & 0,078 & 0,471 & 0,006 \\
\hline EQ3_13-EQ3_07 & $-0,801$ & $-2,332$ & 0,728 & 0,003 \\
\hline EE1_13-EE1_07 & $-0,021$ & $-0,032$ & $-0,011$ & 0,000 \\
\hline L1_13-L1_07 & 0,090 & $-0,073$ & 0,253 & 0,278 \\
\hline O1_13-O1_07 & $-0,009$ & $-0,078$ & $-0,059$ & 0,777 \\
\hline O3_13-O3_07 & 0,026 & 0,019 & 0,033 & 0,000 \\
\hline
\end{tabular}

Fuente: Elaboración propia. 
La rentabilidad económica de los hoteles españoles es menor después de la crisis, al igual que en sectores como el comercio Ramón (2016). Tanto el margen bruto de explotación (RE2) como la rentabilidad económica (RE4) medida como resultados antes de impuestos e intereses en relación con las inversiones han caído como consecuencia de la crisis y, aun cuando el año 2010 es el de la recuperación para el sector del turismo, el aumento del número de turistas y pernoctaciones por sí solo no están permitiendo volver a niveles de rentabilidad previos a la crisis, (gráfico 1).

\section{Gráfico 1: Evolución de la medias de Rentabilidad Económica (RE4), Resultado Bruto de Explotación (RE2), Cifra Neta de Negocio} (CNN), Estancia media y Num. Viajeros. Base 100 año 2007

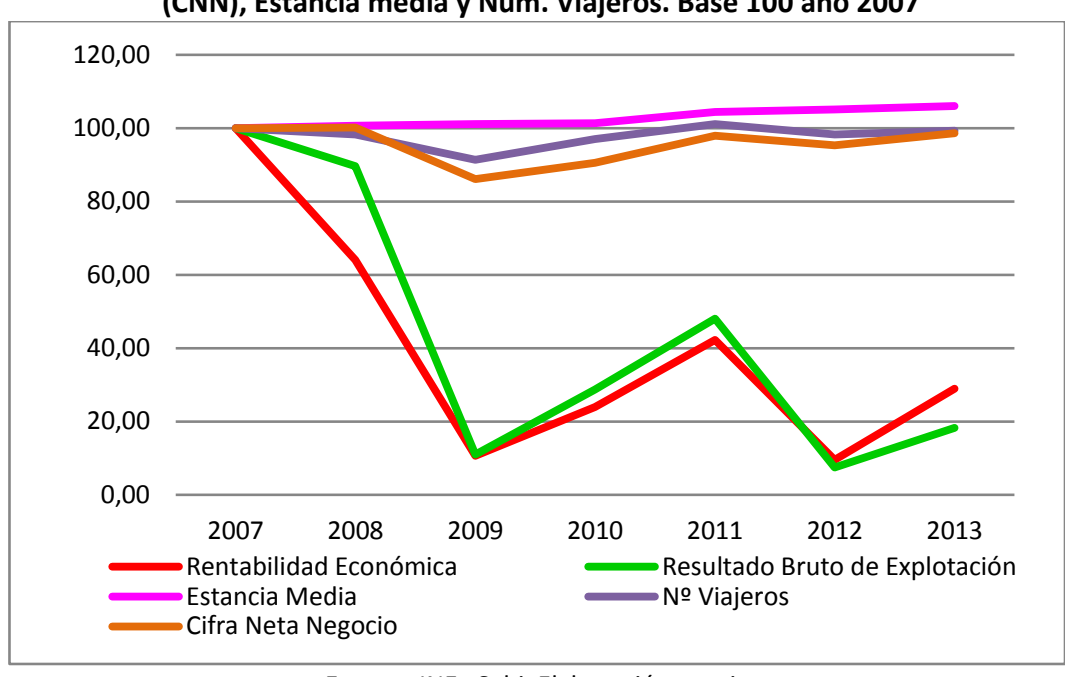

Fuente: INE. Sabi. Elaboración propia.

Durante el 2009, la Cifra Neta de Negocio cayó más intensamente que el número de viajeros y que la estancia media pero, a partir de 2010, los ingresos se recuperan y en 2013 alcanzan los niveles precrisis. En contraposición, la rentabilidad económica y el margen bruto de explotación se desplomaron entre 2008 y 2009 y, después de la crisis, ambos ratios siguen estando en menos del $70 \%$ del valor que había obtenido antes de la crisis. La actividad hotelera ha perdido durante la depresión gran parte de su capacidad para generar recursos dados sus niveles de inversión aunque el comportamiento de la rentabilidad se ha debido a distintos motivos en distintos años.

Así, durante los años 2008 y 2009, la reducción del número de viajeros y de estancia media llevó a una infrautilización de inversiones y por tanto, caídas de la rentabilidad. Por el contrario, a partir de 2010 y con la recuperación de la demanda, la existencia de unos precios demasiado bajos y unos elevados gastos fijos de producción están en la base de esta baja rentabilidad. No obstante, es previsible que conforme siga aumentando la demanda, los precios también lo harán, lo que permitirá que las inversiones vayan recuperando los niveles de rentabilidad perdida.

El bajo margen bruto de explotación abunda en este sentido, el beneficio que se obtiene por cada unidad monetaria de ventas sigue estando muy por debajo del alcanzado antes de la crisis: los aumentos de los ingresos se han visto absorbidos, por un lado, por incrementos en los costes operativos, fundamentalmente la mano de obra y energéticos, y por otro lado, por la existencia de unos precios medios menores, lo que ha contribuido netamente a la disminución de los resultados de explotación y por ende, de la rentabilidad.

Desde la perspectiva de la solvencia, los hoteles españoles que han superado el shock de la crisis muestran un alto, y creciente, nivel de solvencia económica (REnd1). La combinación de menor deuda e incremento del activo con fondos propios adicionales permite ofrecer unas elevadas y crecientes garantías de recuperación para acreedores, lo que es un buen punto de partida para nuevas necesidades de financiación ajena.

La otra cara de la moneda es el alto nivel de endeudamiento (EQ3) del que partían los hoteles españoles antes de la crisis. La expansión del crédito como consecuencia de la política de bajos tipos de interés estimuló a los hoteles, y a otros sectores Fernández y Ramón (2013) a endeudarse durante el primer quinquenio del milenio. Además, la predominancia de grandes cadenas hotelera en el sector, con una posición dominante frente a sus acreedores, puede haber provocado que en el conjunto de la muestra se den unos valores de endeudamiento muy por encima del máximo teórico recomendable. No obstante, a lo largo de la crisis, se ha producido una intensa reestructuración de las fuentes de financiación, reduciendo la ajena e incrementando los recursos propios, lo que va en sintonía con la mejora de la solvencia.

Respecto a la estructura económica apenas ha variado durante la crisis. La relación entre activos corrientes y total activo es pequeña, lo que indica que la mayor parte de inversiones de los hoteles van destinadas a adquisición de bienes de capital y de larga duración, lo cual es lógico a tenor su propia actividad principal: la oferta de alojamiento hotelero se basa, en muchas ocasiones, en la inversión en edificios.

Los ratios (L1) indican que los hoteles españoles tienen una holgada liquidez, lo que permite mostrar una buena sincronización entre la corriente de cobros y pagos inmediatos. Además, teniendo en cuenta el $p$-valor, aceptamos la hipótesis nula de igualdad de medias, lo que expresa que no han existido cambios significativos en los niveles de liquidez como consecuencia de la crisis. Los hoteles españoles pueden hacer frente a sus deudas a corto plazo sin incurrir en descubiertos ni demoras. Algo parecido ocurre con el fondo de maniobra (01) que se mantiene inmóvil y en valores negativos tanto antes como después de la crisis: el sector hotelero 
español se está beneficiando de una financiación espontánea al cobrar al contado y pagar a plazos.

El leve, pero estadísticamente significativo, aumento del ratio operativo (O3) indica una pérdida de productividad de la mano de obra fundamentada, básicamente, en el mantenimiento de las plantillas mientras se reducen las tarifas de precios. No obstante, parece razonable suponer que, conforme se recupere la economía y los precios, la gestión de la plantilla laboral será más eficiente y por tanto mejorará la productividad.

La tabla 4 representa los la capacidad que tienen algunas macromagnitudes de explicar la evolución de la Cifra Neta de Negocio (CNN) en el modelo de regresión después de haber realizado un procedimiento de selección de variables explicativas "hacia atrás".

Tabla 4 - Peso de las macro-magnitudes sobre la evolución de Cifra Neta de Negocio

\begin{tabular}{|l|l|l|l|l|l|l|}
\hline \multicolumn{2}{|c|}{ Modelo } & \multicolumn{1}{|c|}{$\begin{array}{c}\text { Sig. de la } \\
\text { regresión } \\
(\mathbf{p})\end{array}$} & $\begin{array}{c}\text { Coeficientes } \\
\text { tipificados }\left(\boldsymbol{\beta}_{i}\right)\end{array}$ & Sig. & R-cuadrado & $\begin{array}{c}\text { R-cuadrado } \\
\text { corregida }\end{array}$ \\
\hline 1 & Regresión & 0.017 & & & \\
\hline \\
\hline
\end{tabular}

Fuente: elaboración propia.

Al finalizar el proceso de selección de variables, quedó excluida como factor explicativo de la evolución de la CNN, el Número de Viajeros Españoles (Ve), ya que su nivel de significación era superior al 0.05. Por el contrario, la Renta Neta Disponible por Habitante (Yd) y, sobre todo, el número de Viajeros Extranjeros $(\mathrm{Vx})$ mostraban una alta capacidad de influencia (Sig <0.05).

El Modelo 2, es el que mejor explica la evolución de la CNN de los hoteles españoles y además, es el más consistente al ofrecer una significatividad de la regresión próxima a cero $(p=0.003)$ y menor que la del Modelo 1. Matemáticamente, quedaría expresado por (4).

$$
\mathrm{CNN}_{\mathrm{i}}=\alpha+\beta_{1} \mathrm{Y}_{\mathrm{d}}+\beta_{3} \mathrm{~V}_{\mathrm{x}}
$$

Sustituyendo los valores de cada $\beta$ tendríamos (5)

$$
\mathrm{CNN}_{\mathrm{i}}=\alpha+1,09 \mathrm{Y}_{\mathrm{d}}+1,35 \mathrm{~V}_{\mathrm{x}}
$$

Las variaciones en los ingresos hoteleros vienen explicadas por variaciones en el mismo sentido tanto de la Renta per Cápita como del Número de Viajeros Extranjeros. Ahora bien, dado que $\beta_{3}$ es mayor que $\beta_{1}$, podemos decir que, de las dos variables explicativas, la $V_{x}$, es la que mayor importancia relativa tiene. Así, manteniendo constante el nivel de Renta Neta Disponible por Habitante, se puede decir que aumentos en una unidad en el Número de Viajeros Extranjeros provocarán aumentos de la Cifra Neta de Negocio en 1,35 veces.

Los ingresos hotelero y por extrapolación la demanda, mantienen una elasticidad "demanda-renta" positiva con un coeficiente multiplicador $\beta_{1}=1,09$ y que puede fluctuar entre el 1,04 y el 2,03. Pero la elasticidad "demanda-número de viajeros extranjeros" es mayor. De ahí, que la crisis económica se iniciara con caídas en los ingresos de los hoteles más rápidas que las pérdidas de $\mathrm{PIB}_{\mathrm{pm}}$ y de Renta per Cápita, pero en 2010, cuando las macro-magnitudes se desplomaron como consecuencia de la crisis de la deuda soberana en España, los ingresos netos de los hoteles españoles, contra lo que se podría pronosticar, iniciaron una intensa recuperación, alcanzando en 2013 los niveles de ingresos pre-crisis (Gráfico 2).

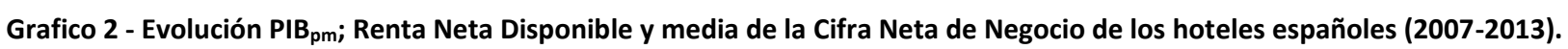
Base 100 año 2007

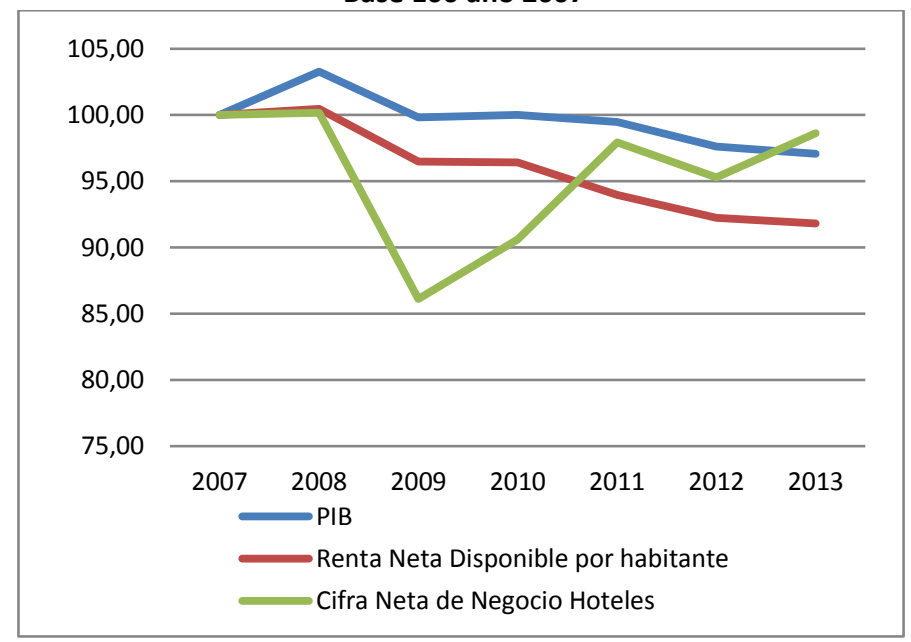

Fuente: INE. SABI. Elaboración propia. 
La inestabilidad política de algunos destinos Mediterráneos junto con el alto grado de competitividad en calidad y precios de la industria hotelera española ha propiciado que los turistas de países sin crisis, prefiriesen España para sus vacaciones. La apuesta del sector de anteponer la supervivencia a la rentabilidad a corto plazo ha atraído a más turistas ingleses y alemanes. Lo que ha permitido que los ingresos del sector crezcan independientemente a la prosperidad de la economía nacional y, aunque sin recuperar toda la rentabilidad perdida, la industria hotelera española está creando riqueza y empleo, produciendo así un efecto anti-cíclico en la economía nacional.

\section{Conclusiones}

El objetivo de nuestro trabajo era ver si la estrategia de supervivencia sectorial a la actual crisis había dejado, o no, huella en los Estados Contables de los hoteles y si esos cambios han evolucionado al mismo ritmo que lo hacían la economía del país.

Del análisis de los ratios económico-financieros se desprende que solo los ratios relacionados con la liquidez y la estructura económica se han mantenido sin modificaciones como consecuencia de la crisis. El resto han sufrido cambios estadísticamente significativos antes y después de la crisis, lo que nos pone sobre la pista de que los hoteles españoles han desarrollado estrategias de supervivencia que tienen su reflejo en la evolución de los Estados Financieros.

En este sentido hemos visto que, pese al crecimiento de la Cifra Neta de Negocio, tanto la rentabilidad económica como el margen bruto de explotación, en 2013, sigue siendo menos de la mitad de lo que fueron antes de la crisis. La mayor ocupación registrada desde 2010 ha venido de la mano, por una parte, de unos aumentos de costes tanto laborales como energéticos y, por otra parte, de unos precios medios menores. La industria hotelera española ha sacrificado parte de su propia rentabilidad para sobrevivir, de ahí que se pueda decir que el mayor número de pernoctación no es una garantía de mejora de la rentabilidad empresarial. No obstante, la recuperación de la cifra neta de negocios es el primer elemento indicador de cambio de tendencia para las resultados de los hoteles, lo que nos lleva a aceptar nuestra $\mathrm{H} 1$ : A pesar de que las pernoctaciones empiezan a recuperarse a partir de 2010, los efectos de la crisis han sido tan intensos y la industria hotelera ha necesitado contener tanto los precios que, en 2013, el número de pernoctaciones alcanzó el de pre-crisis, pero las rentabilidades económicas no. Es necesario, por tanto, incentivar el interés por las pernoctaciones y reestablecer una política de costes y precios más rentable.

También hemos visto que las empresas del sector mantienen un comportamiento anti-cíclico en relación con las variables macroeconómicas como el $\mathrm{PIB}_{\mathrm{pm}}$, o la Renta Neta Disponible por Habitante. Mientras que la economía nacional sigue cayendo, la Cifra Neta de Negocio de la industria hotelera inicia una intensa remontada desde 2010 y, en 2013, alcanza los valores pre-crisis. El sector hotelero ha sabido aprovechar la coyuntura internacional y, renunciando a altas tasas de rentabilidad, ha sido capaz de desligarse del mercado nacional y atraer a más turistas internacionales, sobre todo, los procedentes de países donde no hay crisis.
Desde esta perspectiva, hemos aproximado, a través de un modelo de Regresión Lineal Múltiple, las macro-magnitudes que mayor influencia tienen sobre la evolución de los ingresos del sector. Del análisis hemos apreciado que, aunque existe una elasticidad demanda-renta positiva de 1,09 con un margen de error del 5\%, la variable que más influye en la evolución de los ingresos sectoriales es el Número de Viajeros Extranjeros con un coeficiente multiplicador de $\beta_{3}=1,35$ con un intervalo de confianza del $95 \%$.

La menor dependencia del sector hotelero al mercado nacional, y por tanto, a la evolución de la economía española, avalaría la tesis de que a pesar de tener una elasticidad demanda-renta positiva, la contracción de la industria hotelera no ha sido tan intensa como el volumen de renta, de ahí que aceptemos la $\mathrm{H} 2$ : Aunque la demanda hotelera mantiene una elasticidad demanda-renta positiva, durante este periodo de crisis, ha seguido una evolución dispar a la de la renta neta disponible o el PIB contrayéndose en menor medida. El sector hotelero ha actuado de manera anticíclica para la economía nacional. Su autonomía respecto a la economía nacional le permite tener capacidad de crear renta, riqueza y empleo, incluso en las fases depresivas del ciclo económico, lo cual debería de ser tenida en cuenta y valorado desde las distintas administraciones a la hora de elaborar sus políticas económicas.

Por otra parte, hemos comprobado que se partía de unos elevados niveles de endeudamiento pero que las empresas hoteleras se han esforzado en capitalizarse. Los aumentos de recursos propios orientados a disminuir la dependencia en la financiación ajena han hecho reducir los pasivos e iniciar un proceso de desapalancamiento, lo que ha contribuido a mejorar una solvencia que ya partía de una sólida posición. Por tanto, es aceptable decir que la crisis ha obligado a reestructurar el nivel de endeudamiento del sector, admitiendo así H3: A pesar de que el volumen de negocio de las empresas hoteleras parece que se haya contraído menos que la economía española durante la crisis, la dificultad para acceder al crédito y su encarecimiento ha originado un efecto de desapalancamiento y de recapitalización de los fondos propios.

Pese a las limitaciones del trabajo en el que sólo se ha considerado un periodo de 5 años, se han descartado las microempresas y no se han clasificado las empresas de la muestra podemos decir que, en general, la estrategia de supervivencia a la crisis ha dejado huella en los Estados Financieros de los hoteles españoles. Y, aunque la situación económico-financiera de partida era consistente, el shock económico hizo que la liquidez pero sobre todo, la solvencia y el desapalancamiento financiero, mejorasen. Se podría decir que la crisis ha provocado que la industria hotelera española se esfuerce y perfeccione una estructura económicofinanciera que, de primeras era sólida. Ahora bien, por el camino resta recuperar la rentabilidad perdida. La preferencia de la supervivencia frente a la rentabilidad ha permitido al sector independizarse del mercado nacional y crecer más que éste, produciendo un deseado efecto anti-cíclico en la economía española, no obstante, habría que hacer esfuerzos en reestablecer niveles de rentabilidad similares a los de pre-crisis.

A la luz de todas estas evidencias, creemos que el presente trabajo aporta información relevante sobre un sector económico, que contribuyen significativamente a la creación de PIB, a la generación de empleo y al equilibrio de la balanza de pagos. Ahora 
bien, estos resultados podrían estar condicionados tanto a la muestra seleccionada como al país como a los métodos de análisis utilizados. Pensamos que la comparación con otros sectores igualmente importantes para la economía española, así como la comparación del sector hotelero a escala internacional y por supuesto, la introducción de nuevas técnicas de análisis como las redes neuronales, podrían arrojar nuevos e interesantes resultados que valoramos como posible futuro objeto de estudio.

\section{Referencias}

Álvarez García, J., Fraiz Brea, J.A. \& Del Río Rama, M.C. (2012). Análisis de las motivaciones para certificar la marca $Q$ de calidad turística. Sector se alojamiento. Investigaciones Europeas de Dirección y Economía de la Empresa, 18(1), 101-121.

Altmant, E.I. (1968). Financial ratios, discriminant analysis and the prediction of corporate bankruptcy. The Journal of Finance, 23(4), 589-609. Altmant, E.I. \& Sabato, G. (2007). Modelling credit risk for SMS: Evidence from the US market. Avacus, 43(3), 332-357.

Amat Salas, O. (2000). Análisis de estados financieros: fundamentos y aplicaciones. Barcelona: Gestión 2000.

Archel Domench, P., Lizárraga Dallo, F. \& Sánchez Alegría, S. (2008). Estados contables. Elaboración, análisis e interpretación. Madrid: Pirámide.

Aurioles, J. (2011). Crisis económica y cambios en el turismo". Mediterráneo Económico 20, 99-113.

Benishay, H. (1971). Economic information in financial ratio analysis: A note. Accounting and Business Research, 1(2), 174-179.

Booth, L., Aivazian, V. A., Demirguc-Kunt, A. \& Maksimovic, V. (2001). Capital structures in developing countries. Journal of Finance, 56(1), 87130.

Cohen, E. \& Neal, M., (2010). Coinciding crises and tourism in contemporary Thailand. Current Issues in Tourism, 13(5), 455-475.

Chow-Chua, C., Goh, M. \& Wan, T.B. (2003). Does ISO 9000 certification improve business performance? International Journal of Quality \& Reliability Management, 20, 936-953.

Dahles, H. \& Susilowati, T.P. (2015). Business resilience in times of growth and crisis. Annals of Tourism Research, 51, 34-50.

Demirgüç-Kunt, A. \& Maksimovic, V. (1999). Institutions, financial markets and firm debt maturity. Journal of Financial Economics, 54, 295-336.

Esteo Sánchez, F. (1998). Análisis contable de la rentabilidad empresarial. Madrid: Centro de Estudios Financieros.

Fernández, F. (2010). Crisis financiera, crisis real y competitividad de la economía española. Cuadernos de pensamiento político, 37, 37-60.

Fernández Alles, M.T. \& Cuadrado Marqués, R. (2011). La Responsabilidad Social Empresarial en el Sector Hotelero: revisión de la literatura científica. Cuadernos de Turismo, 28, 47-57.

Fernández Daza, E. \& Ramón Dangla, R. (2013). El efecto de la crisis en dos sectores importantes de cooperativas valencianas. Similitudes y diferencias con sus homologas en las sociedades de capital. Ciriec-España. Revista de Economía Pública, Social y Cooperativa, 79, 219-242.

Fernández, M.A. \& Becerra, R. (2015). An analysis of Spanish hotel efficiency. Cornell Hospitality Quartely, 53(3), 248-258.

Foster, (1986). Financial Statement Analysis. Englewood Cliffs: NJ Prentice Hall.

Gallizo, J.L. y Salvador, M. (1997). Análisis sectorial de las fuerzas equilibradoras de ratios financieros. Revista Española de Economía, 14 (2), 229-250.

García Rodríguez, F.J. \& Armas, D.M. (2007). Relation between socialenvironmental responsability and performance in hotel firms. International Journal of Hospitality Management, 26, 824-839.

Garrido Miralles, P. \& Íñiguez Sánchez, R. (2008). Los ratios en el nuevo Plan General de Contabilidad. En G. López Espinosa (Cood), Fundamentos teóricos y prácticos del nuevo Plan General Contable. Pamplona: Thomson Aranzadi.

Garrido Miralles, P. \& íñiguez Sánchez, R. (2012). Análisis de Estados Contables. Elaboración e interpretación de la información financiera. Madrid: Pirámide.
Gémar, G., Moniche, L. \& Morales, A.J. (2016). Survival analysis of the Spanish hotel industry. Tourism Management, 54, 428-438.

Gill de Albornoz, B. \& Giner, B. (2013). Predicción del fracaso empresarial en los sectores de construcción e inmobiliario: Modelos generales versus específicos. Universia Business Review, 39 (Tercer Trimestre), 118-131.

Godoy López, L. (2004). Preparación y análisis de estados financieros. Bilbao: Desclée de Brouwer.

González, V. \& González, F. (2008). Influence of bank concentration and institutions on capital structure: new international evidence. Journal of Corporate Finance, 14, 363-375.

González, C. \& Jareño, F. (2014). Financial analysis of the main hotel chains of the Spanish tourism sector. Regional and Sectoral Economic Studies, 14(2), 91-108.

Gonzalo Angulo, J.A., Pérez García, J. \& Serrano García, R. (2000) Contabilidad Superior. Análisis Económico-Financiero y Patrimonial. Madrid: Instituto de Auditores-Censores Jurados de Cuentas de España.

Hajibaba, H., Gretzel, U.,Leisch, F. \& Dolnicar, S. (2015). Crisis-resistant tourists. Annals of Tourism Research, 53, 46-60.

Hanousek, J. \& Shamshur, A. (2011). A stubborn persistence: Is the stability of leverage ratios determined by the stability of the economy? Journal of Corporate Finance, 17(5), 1360-1376

hotelsmag.com July/August 2014. HOTELS 325

Iranzo Martín, J.E. (2003). La demanda de turismo. En Irazo, J.E.: La estructura económica de los mercados turísticos (pp. 59-93). Madrid Instituto de Estudios Económicos.

Jiung-Bin, C., Mu-Chen, W. \& Ling-Feng, H. (2013). Strategic planning of optimal resource allocation in response to global financial crisis - a study of international tourist hotels. Applied Economics, (45) 23, 3316-3328.

Jordà, O., Schularik, M. \& Taylor, A. M. (2011). When credit bites back: leverage, business cycles and crises. NBER Working Paper, 17261.

Lado-Sestayo, R., Vivel-Bua, M., Otero-González, L. \& Martorell-Cunill, O (2016). Impact of location on profitability in the Spanish hotel sector. Tourism Management, 52, 405-415.

Lee, C. \& Wu, C., (1988). Expectation formation and financial ratio adjustment processes. The Accounting Review, 2, 292-306.

LEV, B. (1969). Industry averages as targets for financial ratios. Journal of Accounting Research, 7, 290-299.

Lo, A., Cheung, C. y Law, R. (2006). The survival of hotels during disaster: A case study of Hong Kong in 2003. Asia Pacific Journal of Tourism Research, 11(1), 65-80.

Mak, B.L.M. (2011). ISO certification in the tour operator sector. International Journal of Contemporary Hospitality Management, 23, 115130.

Massieu, A. (2004). El sector turístico visto desde la OMT: una reflexión a partir de la coyuntura reciente a nivel mundial. En Uriel, E. y Hernández, R. (Ed): Análisis y tendencias del Turismo, (pp. 19-44). Madrid: Piramide.

Martínez López, A.M. (2009). Innovation in the Spanish tourism industry: factors determining the innovate capacity of the Spanish hotel sector, using the approach of the strategic management process. European Journal of Tourism Research, 2, (2), 194-196.

Miguel, A. De, \& Pindado, J. (2001). Determinants of capital structure: new evidence from Spanish panel data. Journal of Corporate Finance, 7, 77-79.

Murphy, H., Chen, M-M. \& Cossutta, M. (2015). An investigation of multiple devices and information sources used in the hotel booking process. Tourism Management, 52, 44-51.

OMT (2004). Turismo y atenuación de la pobreza. Madrid: OMT

Observatorio de la Industria Hotelera Española. Confederación Española de hotels y alojamientos

OMT (2010). Manual on Tourism and Poverty Alleviation Practical Steps for Destinations.

Ortega, E. y Peñalosa, J. (2012). Claves de la crisis económica española y retos para crecer en la UEM. Banco de España, Documentos ocasionales, Núm. 1201.

Peles, Y. \& Schneller, M., (1989). The duration of the adjustment process of financial ratios. The Review of Economics and Statistics, 62, 527-532.

Pérez, A. \& Rodríguez del Bosque, I. (2015). Estado de la comunicación y de responsabilidad social corporativa en el sector hotelero español. Cuadernos de Turismo, 36, 315-338. 
Pertusa Ortega, E.M., Tari Guillo, J.J., Pereira Moliner, J., Molina Azorin, J.F. \& López Gamero, M.D. (2013). Certificación en calidad, resultados empresariales y estructura organizativa en el sector hotelero español. Intangible Capital, 9(1), 199-224.

Rajan, R. \& Zingales, L. (1995). What do we know about capital structure? Some evidence from international data. The Journal of Finance, 50(5), 1421-1460.

Ramón Dangla, R. (2016). Cuando la crisis te exime. El sector de la distribución y el comercio al por menor español: pequeñas vs grandes empresas. Revista Galega de Economía, Vol 25(1), 23-40.

Ramón, A. (2002). Estrategias de desarrollo en la industria hotelera mundial. Momento Económico, 119, 35-54.

Rivero Torre, P. (1996). Análisis de balances y estados complementarios. Madrid: Pirámide.

Rubio Andrada, L., Alonso Almeida, M.M. \& Rodríguez Antón, J. (2011). Motivations and impacts in the firm and stakeholders of quality certification: evidence from small- and medium-sized service enterprises. Total Quality Management \& Business Excellence, 22, 833-852.

Rubio, G. \& Sogorb, F. (2011). The adjustment to target leverage of Spanish public firms: macroeconomic conditions and distance from target. Revista de Economía Aplicada, 57, 35-63.

Ruíz Gómez, L.M. \& Casado García-Hirschafeld, E. (2011). La actividad turística en la Unión Europea en un entorno de crisis económica y financiera global. Saberes. Revista de estudios jurídicos, económicos y sociales, 9, 4-12.

Sánchez Arroyo, G. (2002). Ejercicios de contabilidad y análisis e interpretación de la información contable. Madrid: Pirámide.

Sharpley, R. (2008). The tsunami and tourism: a comment. Current issues in Tourism, 8(4), 344-349.

Sawilowsky S. (2005). Misconceptions leading to choosing the t test over the Wilcoxon Mann-Whitney $U$ test for shift in location parameter. Journal of Modern Applied Statistical Methods 4(2), 598-600.

Souto, J. (2015). Business model innovation and business concept innovation as the context of incremental innovation and radical innovation. Tourism Management, 51, 142-155.

Torres Bernier, E. (1994). Turismo y desarrollo regional. Papers de Turisme, 14-15, 95-102.

Torres Bernier, E. Ramírez Sánchez, R. y Rodríguez Díaz, B. (2014). La crisis económica en el sector turístico. Un análisis de sus efectos en la Costa del Sol. Revista de análisis Turístico, 18, 11-18.

Tsai, H., Song, H. y Wong, K. (2009). Tourism and hotel competitiveness research. Journal of Travel \& Tourism Marketing, 26(5-6), 522-546.

Urías Valiente, J. (1995). Análisis de estados financieros. Madrid: McGrawHill.

Vila, M., Enz, C., \& Costa, G. (2012). Innovative practices in the Spanish hotel industry. Cornell Hospitality Quarterly, 53(1), 75-85.

Wu, C. \& Ho, S.K. (1997). Financial ratio adjustment: industry-wide effects on strategic management. Review of Quantitative Finance and Accounting, 9, 71-88.

Recibido: 18.04.2016

Reenviado: 24.07 .2016

Aceptado: 25.07.2016 